\title{
Mean Stable Warfarin Doses versus CYP2C9*2 and VKORC11639G>A Genotypes in Sudanese Population
}

\author{
Azza A. M. H. Swar Aldahab*, Abdalla O. Elkhawad, Ahmed S. A. Elsayed and Hanan B. Eltahir \\ University of Medical Sciences and Technology, Faculty of Pharmacy, Department of Pharmacology, Khartoum, \\ Khartoum state, Sudan; azzaswaraldahab@yahoo.com
}

\begin{abstract}
Warfarin is a potent anticoagulant with a confirmed effectiveness when anticoagulation targets are attained, an issue, that is troublesome to reach due to the fact that warfarin has a narrow therapeutic index (NTI), that means they have a narrow window between their effective doses and those at which they produce adverse toxic effects. However, oral anticoagulation throughout genetics recommended a genotype guided dosing, but is it favourable over clinical based dosing? Objectives: To analyze the mean stable warfarin doses attained clinically within CYP2C9*2 and VKORC11639G $>$ A wild-type and variant genotype status in Sudanese patients. Method: Genotyping for the CYP2C9*2 and VKORC1-1639G>A polymorphisms were accomplished with polymerase chain reaction-restriction fragment length polymorphism(PCR-RFLP) technique. The mean stable warfarin dose per genotype was defined as the mean stable warfarin dose related to the stable INR within target range within a genotype of each of CYP2C9*2 or VKORC11639G> Agenes, using Analysis of Variance (ANOVA) as the statistical method. Results: Fifty-three stable patients with wild-type CYP2C9*1*1 genotype had a mean stable warfarin dose of $4.9 \pm 2.1 \mathrm{mg}, 5$ patients who were heterozygous CYP2C9* $1 * 2$ genotype, had a mean stable warfarin dose of $5.0 \pm 0.71 \mathrm{mg}$, and 2 patients were homozygous mutant CYP2CP*2*2 genotype had a mean stable warfarin dose of $4.8 \pm 0.3 \mathrm{mg}$. The results were statistically insignificant, $\mathrm{P}=0.992$.Sixteen unstable patients were of wild-type CYP2C9* $1{ }^{*} 1$ genotype, 40 patients with heterozygous CYP2C9*1*2 genotype, and 4 with homozygous mutant CYP2CP* $2 * 2$ genotype, had mean stable doses of $5.32 \pm 2.9,6.5 \pm 2.7$ and $4.5 \pm 0.71 \mathrm{mg}$ respectively. The result was statistically insignificant, $\mathrm{P}=0.508$. Fifty-two stable patients were having wild-type VKORC1G/G genotype, 3 patients had heterozygous VKORC1G/A genotype and 3 patients had homozygous mutant VKORC1/AA genotype, these patients had mean stable doses of $5.41 \pm 1.63,4.8 \pm 2.19$ and $5.4 \pm 0.99$ mg respectively. The mean warfarin stable dose among homozygous mutant VKORC1A/A genotype was lower than among wild-type and heterozygous genotype profiles. This result was statistically not significant, $\mathrm{P}=0.729$. In the unstable group, 8 patients of wild-type VKORC1G/G genotype, had a mean stable warfarin dose of $7.34 \pm 3.9 \mathrm{mg}, 40$ patients of heterozygous VKORC1G/A genotype had a mean stable warfarin dose of $5.21 \pm 2.76 \mathrm{mg}$, and 10 patients of homozygous VKORC1A/A genotype had a mean stable warfarin dose of $4.3 \pm 1.83 \mathrm{mg}$. The result was statistically insignificant, $\mathrm{P}=0.067$.Conclusion: In our study, as there were no significant differences between warfarin mean stable doses related to different CYP2C9*2 and VKORC11639G>A genotypes, the evidence is not satisfactory to conclude that the conventional use of genotype guided warfarin dosing will correct stable warfarin dose among Sudanese patients.
\end{abstract}

Keywords: Genotype, Stable Dose, Warfarin

\section{Introduction}

As it has been known upto date, genotypes of VKORC11639G $>A$ and CYP2C9*2 are important elements in alterations of warfarin dose, but in an unsatisfactory way as they explain less warfarin dosing complications through patients of African descents than for those of European or Asian descents. The challenge in the clinical implementation of the genetic information about warfarin is due to the scarcity of applicable dosing algorithm that fits for all ethnic groups around the world ${ }^{1}$.Therefore, as there is a question about the utility

${ }^{*}$ Author for correspondence 
of genotype guided warfarin dosing in our Sudanese patients, we directed this work in order to determine whether genotypes of CYP2C9 2 and VKORC11639G $>A$ genes influence the administration of warfarin dosing in Sudanese population? Taking into account that the causative variants have not been confirmed with accuracy in such African populations. Many trials reported that a genotype guided dosing strategy did not make alleviation among patients' clinical outcomes ${ }^{2}$.

Also, another study revealed that a gene based method for warfarin dosing is not better than standardized dosing methods. Despite Africans are generally known for their nearly high mean warfarin doses-these increased warfarin doses could be elucidated by the low frequencies of the variant minor alleles among them and consequently lower heterozygous and homozygous mutant genotypes ${ }^{3}$.

\section{Objective}

To analyze the mean stable warfarin doses attained clinically within wild-type and variant CYP2C9*2 and VKORC11639G >A genotype status in Sudanese patients.

\section{Materials and Methods}

The anticoagulation history and a complete warfarin dosing information were recalled from the patients' medical archives of a hundred and eighteen Sudanese patients on warfarin treatment for long term treatment who were checking in the anti-coagulation clinic at the Sudan Heart Centre, Khartoum, Sudan. The patients were chosen depending on all being Sudanese who were on a constant warfarin treatment.T hey were examined for their warfarin stable doses clinically administered through their different genotype formsof CYP2C9*2 and VKORC11639G $>$ A genes, all patients were characterized as stable and unstable according to their general anticoagulation control. $4 \mathrm{ml}$ of blood were derived from allpatients; DNA was extracted from blood samples by approved methods using the QIAamp DNA Blood Mini Kit (Quiagen) determined for rapid purification of genomic DNA. Genotyping for the CYP2C9*2 and VKORC1-1639G>A polymorphisms were accomplished with polymerase chain reaction-restriction fragment length polymorphism (PCR-RFLP) technique. PCR for CYP2C9*2 and VKORC1-1639G> Awas worked out using PCR technique of Maxime PCR PreMix Kit (i-Taq) for amplification of the DNA material which was fulfilled in a final volume of 20 ul.PCR cyclic conditions constituted of an initial denaturation at $94^{\circ} \mathrm{C}$ for $5 \mathrm{~min}$, followed by 35 cycles of denaturation at $94^{\circ} \mathrm{C}$ for $30 \mathrm{~s}$, annealing at $58^{\circ} \mathrm{C}$ for $30 \mathrm{~s}$ and elongation at $72^{\circ} \mathrm{C}$ for $30 \mathrm{~s}$, finalized by an extension period at $72^{\circ} \mathrm{C}$ for $10 \mathrm{~min}$. The CYP2C9*2 and VKORC1-1639G $>$ APCR product reaction mixture formation is shown in Table 1.

Table 1. Preparation of CYP2C $9 * 2$ and VKORC11639G $>$ A PCR reaction mixtures

\begin{tabular}{lc}
\hline Reaction mixture & Volume \\
\hline Genomic DNA & $2 \mathrm{ul}$ \\
Primers (Forward:10pmol/ul) & $1 \mathrm{ul}$ \\
Primer (Reverse: $10 \mathrm{pmol} / \mathrm{ul})$ & $1 \mathrm{ul}$ \\
Distilled water & $16 \mathrm{ul}$ \\
\hline Total & $20 \mathrm{ul}$ \\
\hline
\end{tabular}

For the RFLP technique, the restriction enzymes were prepared by adding $1 \mathrm{ul}$ of the buffer to $10 \mathrm{ul}$ of distilled water, then we added $2.5 \mathrm{ul}$ of the diluted buffer to $10 \mathrm{ul}$ of PCR product, and $0.5 \mathrm{ul}$ of restriction enzyme and $12 \mathrm{ul}$ of distilled water generating a final reaction volume of $25 \mathrm{ul}$, as shown in Table 2.The PCR product was cut off with the restriction enzyme Msp1 for CYP2C9*2 and Ava11 for VKORC11639G $>$ A gene by adding the volumes shown and left for incubation for 15 minutes at 37 degrees. The products were then fragmented on $2.5 \%$ agarose gel stained with ethidium bromide and visualized under UV light; genotypes frequencies for both genes were calculated along with their corresponding mean stable warfarin doses(Figures 1-4).The mean stable warfarin dose per genotype was defined as mean stable warfarin dose related to the CYP2C9 or VKORC1 genotypes of the stable INR measurements within target range in both stable and unstable patients.

Table 2. Preparation of CYP2C9*2 and VKORC11639G > A RFLP

reaction mixtures

\begin{tabular}{lc}
\hline Reaction mixture & Volume \\
\hline Diluted buffer & $2.5 \mathrm{ul}$ \\
Restriction enzyme & $0.5 \mathrm{ul}$ \\
PCR product & $10 \mathrm{ul}$ \\
Distilled water & $12 \mathrm{ul}$ \\
\hline Total & $\mathbf{2 5 u l}$ \\
\hline
\end{tabular}


The genotype specified mean stable warfarin dose was calculated as:

Mean stable warfarin dose/genotype $=$ No.of warfarin stable dosesper genotype/total number of doses per genotype.

\section{Results}

Mean stable warfarin doses versus $\mathrm{CYP}_{2} \mathrm{C}^{*} 2$ and VKORC1-1639G $>$ A genotypes in stable and unstable groups:

- $\mathrm{P}=0.992$ using Analysis of Variance (ANOVA) as the statistical method.

In CYP2C9* ${ }^{*} 1$ wild-type homozygous genotype, the mean warfarin stable dose was $4.98 \pm 2.31$, in CYP2C9 ${ }^{*} 1{ }^{*}$ heterozygous genotype $5.0 \pm 0.17$, in CYP2C9 $2 * 2$ :homozygous mutant genotype $4.83 \pm 0.29$. Bars and Error bars represent mean and standard deviation respectively (Figure 1).

- $\mathrm{P}=0.729$ using Analysis of Variance (ANOVA) as the statistical method.

CYP2C9*1*1 wild type homozygous genotype the mean warfarin stable dose was $5.32 \pm 2.97$, in CYP2C9* ${ }^{*}{ }^{*} 2$ heterozygous genotype $6.5 \pm 2.65$, in CYP2C9*2*2:homozygous mutant genotype $4.5 \pm 0.71$. Bars and Error bars represent mean and standard deviation respectively (Figure 2 ).

- $\mathrm{P}=0.508 \mathrm{using}$ Analysis of Variance (ANOVA) as the statistical method.

In VKORC1/GGwild-type genotype mean stable warfarin dose was 5.41 \pm 1.63.In VKORC1/ GA heterozygous genotype4.76 \pm 2.19 , inVKORC1AA homozygous mutant genotype 5.4 \pm 0.99 . Bars and Error bars represent mean and standard deviation respectively (Figure 3).

- $\mathrm{P}=0.067$ using Analysis of Variance(ANOVA) as the statistical method.

In VKORC1/GGwild-type genotype mean stable warfarin dose was 7.38 \pm 3.89 ,In VKORC1/ GA heterozygous genotype $5.21 \pm 2.76$, inVKORC1AA homozygous mutant genotype4.3 \pm 1.83 .Bars and Error

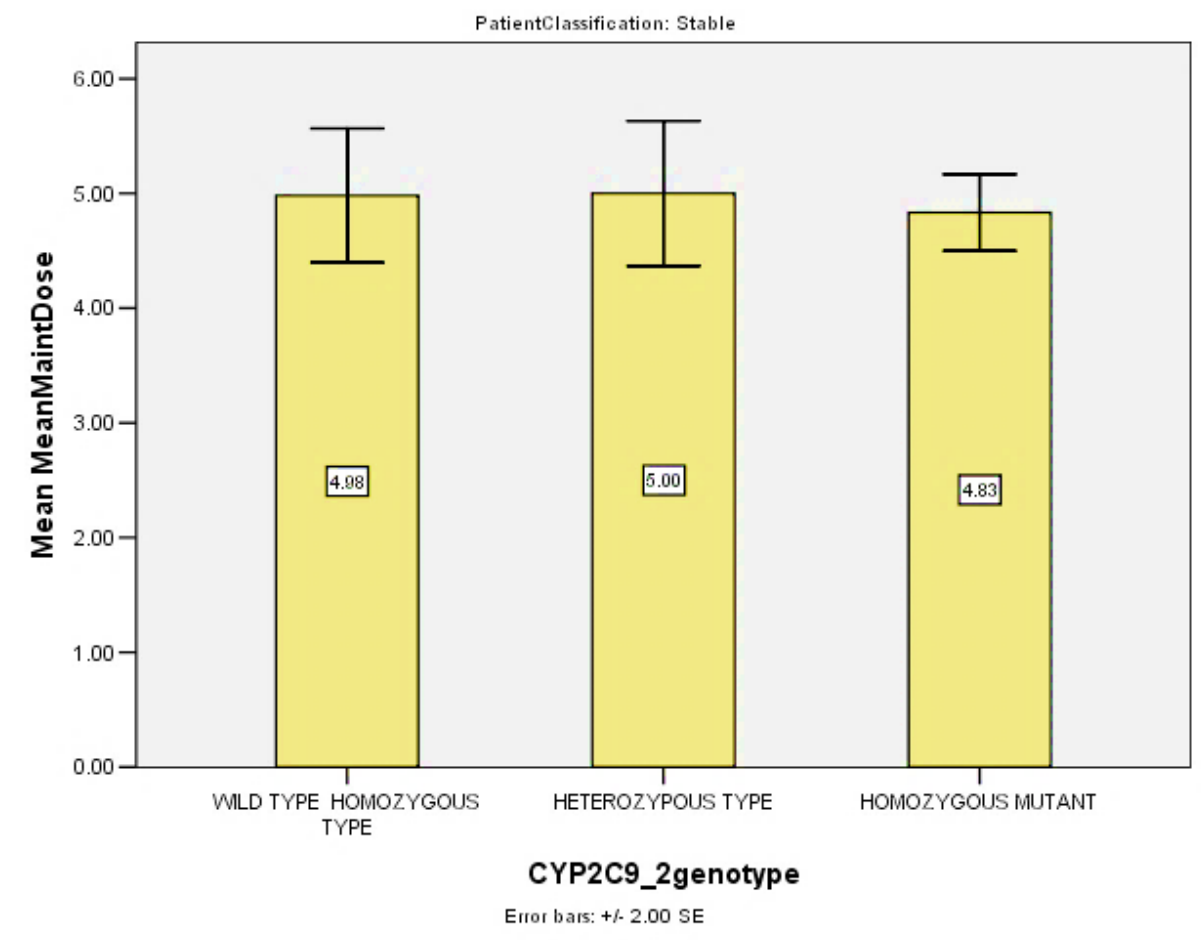

Figure 1. Mean stable warfarin dose mg/day in stable INR group of Sudanese patients on long term warfarin therapy with reference to carrier status for polymorphism in CYP2C9*2. 


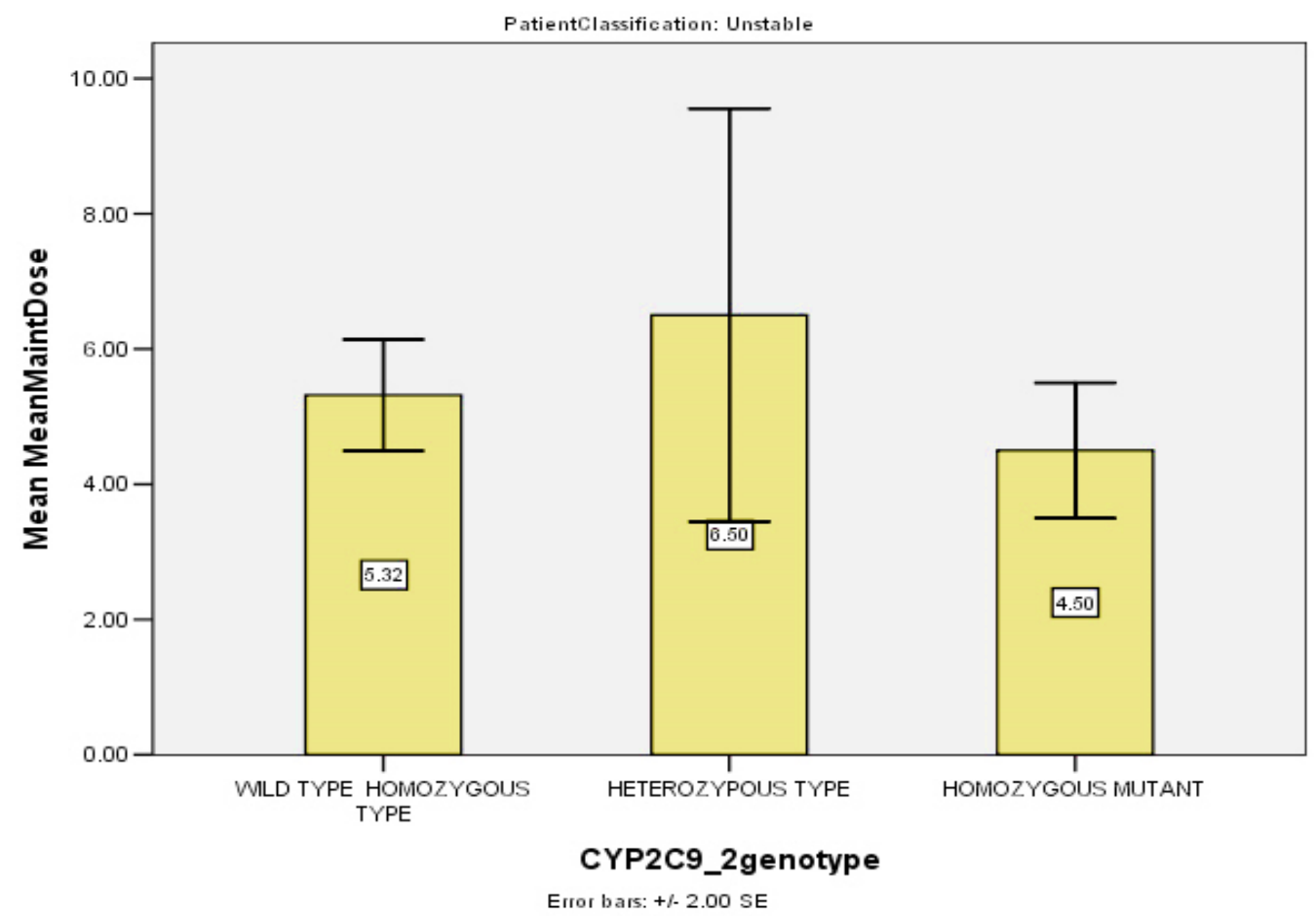

Figure 2. Mean stable warfarin dose ( $\mathrm{mg} /$ day), in unstable INR group of Sudanese patients on long term warfarin therapy with reference to carrier status for polymorphism in CYP2C9*2.

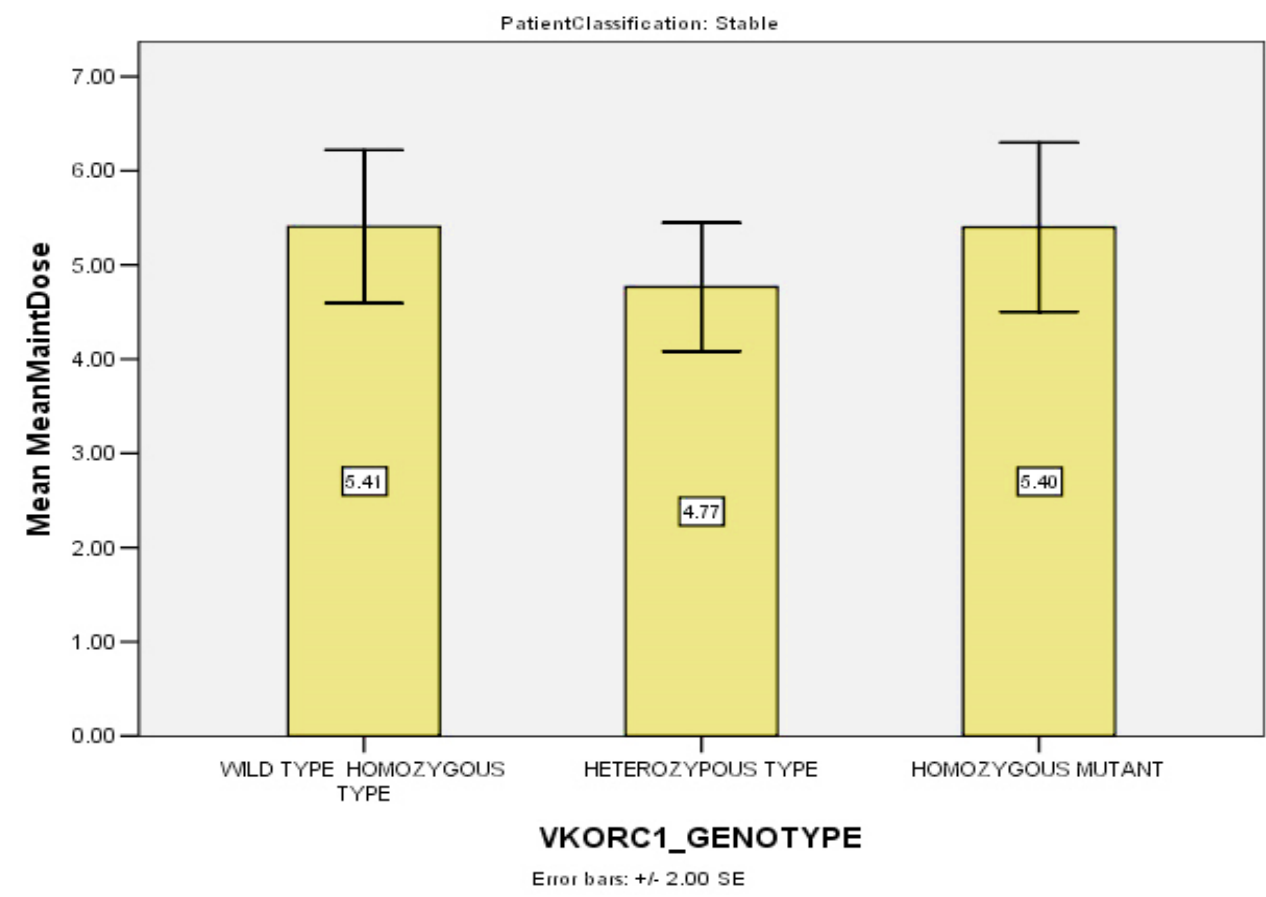

Figure 3. The genotypes and the warfarin stable dose (mg/day) among the stable INR group of Sudanese patients on long term warfarin therapy with reference to carrier status for polymorphism in VKORC1-1639G $>$ A. 


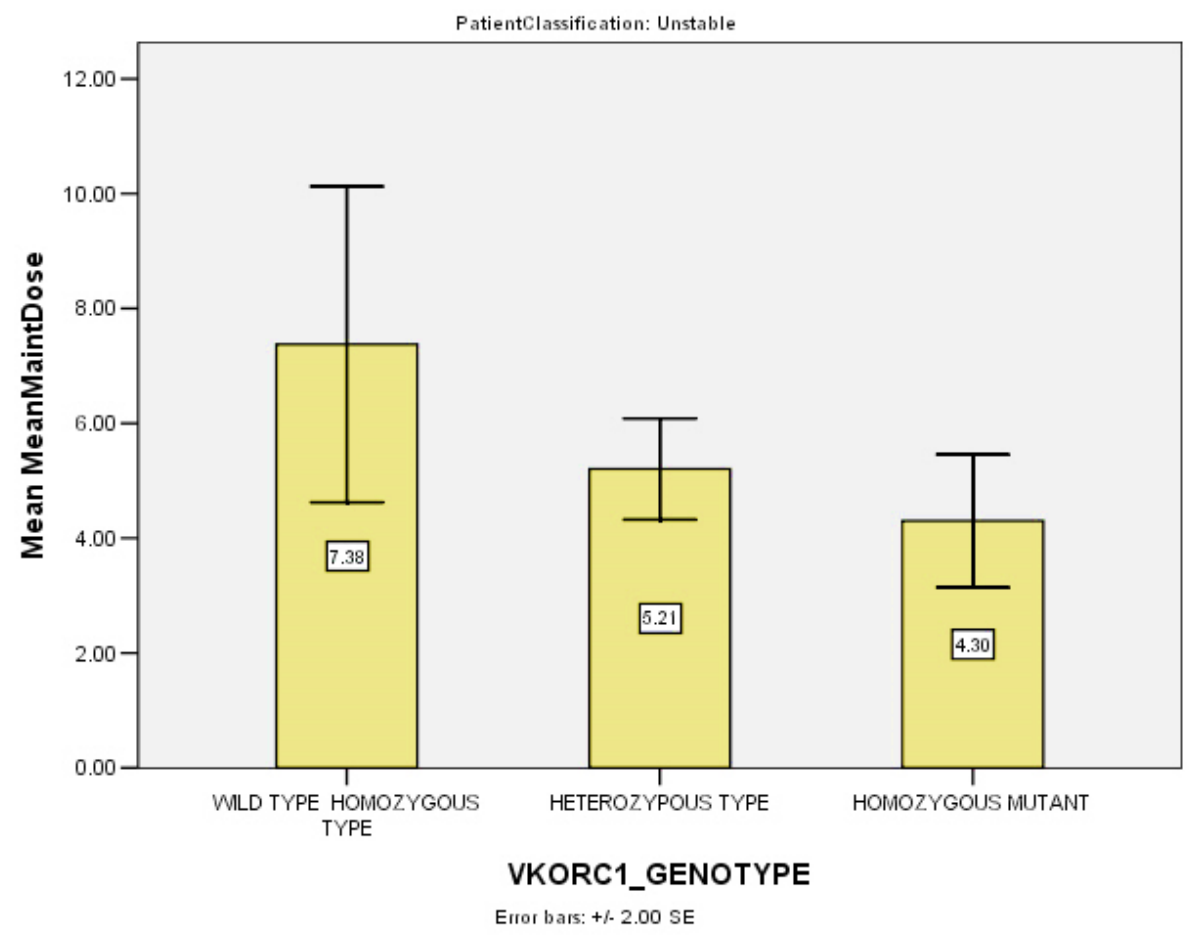

Figure 4. The genotypes and the stable warfarin dose(mg/day) among the unstable INR group of Sudanese patients on long term warfarin therapy with reference to carrier status for polymorphism in VKORC11639G>A, N=118.

bars represent mean and standard deviation respectively (Figure 4).

\section{Discussion}

Does providing of a genotype dosing schedule fundamental for prescribing warfarin dose in Sudanese patients who are on long term warfarin treatment? To answer such a question, we must interpret the genotype dependant warfarin dosing in Sudan. Depending on the literature, knowledge of CYP2C9 and VKORC1 genotypes had reduced warfarin dosing variability. In a meta-analysis, genotype guided dosing did not result in less warfarin dose variability, therefore, in order to study if Sudanese patients clinical doses matched the recommended genotype guided dosing or not, we had to calculate patients' mean stable warfarin doses related to their different genotypes, in order to look if there is a match to the recommended genotyping guided dosing, so as to be used in the future routine dosing programs if the results were promising ${ }^{4}$.
Although some studies did not accomplish a significant association between CYP2C9 ${ }^{*} 2$ and VKORC1 genotypes and warfarin doses in many populations including Africans. The inadequacy in finding such association might be due to the low minor allele frequency of the variant alleles of both CYP2C9 2 and VKORC11639G>A genes, or to the poor number of studies using small samplesizes carried in Africans 5.53 patients with wild-type CYP2C9 ${ }^{*} 1^{*} 1,5$ patients were heterozygous CYP2C9 ${ }^{*} 1^{*} 2$ genotype, and 2 patients were homozygous mutant CYP2CP* $2 * 2$ genotype(Figure 1 ). The mean stable warfarin doses were comparable and without significant difference $P=0.992$. This inconsequential difference could be justified by a study that found a less frequent CYP2C9 variant allele among Africans to the contrary of the high frequency among Europeans, so it alters the warfarin dose in Europeans but not in Africans.This study supports our results regarding different CYP2C9 genotypes and warfarin doserepresentation ${ }^{6}$.

The moderately high dose of $6.52 \mathrm{mg}$ in the heterozygous genotype of $\mathrm{CYP} 2 \mathrm{C} 9^{*} 1^{*} 2$ suggested that the 
presence of the variant allele is not affecting the mean stable warfarin dose of carrier patients. On the other hand, we observed that carriers of the normal genotype of CYP2C9* $1^{*} 1$ had a higher dose of $5.32 \pm 2.9$ compared to the smaller mean dose needed by the variant allele genotype carriers of CYP2C $9^{*} 2^{*} 2$, which was $4.5 \pm 0.71 \mathrm{mg}$, this observation complies to the study that mentioned the carriers of ${ }^{*} 2$ variant alleles need a lower warfarin dose, but without a significant difference (Figure2)7.

Also in another study, 14 individuals randomized to genotyping guided dosing of 1299(1.1\%) patients had thrombotic events compared to 16 of $1287(1.2 \%)$ patients randomized to clinical dosing. Accordingly, genotype reference to warfarin dosing profile illustrated that the genetic test did not result in significant clinical progress and safe anticoagulation outcomes ${ }^{8}$.

Fifty-two stable patients were having wild-type VKORC1G/G genotype, 3patients had heterozygous VKORC1G/A genotype, and 3 patients had homozygous mutant VKORC1/AA genotype. The mean warfarin stable dose among homozygous mutant VKORC1/AA genotype was lower than among wild-type and heterozygous genotype profiles. This result was statistically insignificant, $\mathrm{P}=0.729$ (Figure3).

In the unstable group, 8 patients of wild-type VKORC1G/G genotype, 40 patients of heterozygous VKORC1G/A genotype, and 10 patients of homozygous VKORC1A/A genotype. The result is statistically insignificant, $\mathrm{P}=0.067$ (Figure 4).

The mean warfarin stable dose among homozygous mutant VKORC1/AA genotype was lower than among wild-type and heterozygous genotype profiles. The mean stable warfarin dose was not statistically lower in stable and unstable groups in VKORC11639/AA compared to GG and GA genotype profiles, this observation is in concordance with other studies that found carriers of VKORC1 variant alleles had a tendon to lower warfarin dose requirement ${ }^{9}$.

We observed that the high dose of $7.34 \pm 3.9 \mathrm{mg}$ in the VKORC1GG genotype which complies with the studies that had suggested Africans as populations that need a high warfarin dose in order to gain safeanticoagulation 10 . Also, meta-analysis based on 17 studies reported that compared to VKORC1-1639/AA carriers, VKORC1/GA and GG carriers required a higher mean daily warfarin dose11. Atrend which had also been observed in patients carrying heterozygous versus homozygous mutant subjects who were prescribed higher doses of $6.9 \mathrm{mg}$ than those carrying wild-type genotypes that required a dose of $5.2 \mathrm{mg} 12$.The small dose of $4.3 \pm 1.83 \mathrm{mg}$ in the homozygous genotype of VKORC1AA also confined to previous studies which revealed that polymorphism in VKORC11639G $>A$ gene could lead to small dose requirement13. Although the mean stable doses studied didnot differ significantly between the wild-type and variant genotypes of both genes, this could mean that genetic information did not show an effect in warfarin dosing. It is difficult to analyse individuals from African descents compared to other races, due to the extensive genetic population structure. Although it was found that there is a limited utility of polymorphism effects on the dose among blacks. Therefore, great inter individual dose variability could be found among such populations 14 .

\section{Conclusion}

In our study, the evidence is not satisfactory to conclude that the conventional use of CYP2C9*2 and VKORC11639G $>$ A genotypes guided warfarin dosing do correct stable warfarin dose among Sudanese patients. In addition to the very few data about warfarin genotype dependent dosing in our country, and the few recommendations have been done before in this issue.

\section{References}

1. Johnson JA. Ethnic differences in cardiovascular drug response.Circulation. 2008; 118:1383-93.

2. Limdi NA, Brown TM.Race influences warfarin dose changes associated with genetic factors.Blood. 2015; 126:39-545.

3. Kimmel SE.Pharmacogenetics versus clinical algorithm for warfarin dosing.New England Journal of Medicine. 2013; 369:2283-93.

4. Aithal GP,Day CP.Association of polymorphism in CYP2C9 with warfarin dose requirement and risk of bleeding complications. Lancet. 1999;353:717-9.

5. Moyer TP, DennisJ. Warfarin sensitivity genotyping: a review of literature and summary of patients experience. Mayo Clinic Proceedings.2009; 84(12):1069-94.

6. Limdi NA,GoldsteinJA.Influence of CYP2C9 genotype on warfarin dose among African American and European Americans.Personalized Medicine. 2007;4(2):157-69.

7. Yin $T$, MiyataT.Warfarin dose and pharmacogenomics in oral anticoagulation therapy.BrJ Clin Pharmaol.2006; 61(7):99-111.

8. Sterglopoulos K, Brown D.Genotype guided versus clinical 
dosing of warfarin and its analogues. JAMA InternMed. 2014; 174(8):1330-8.

9. Kawr A, Kan F. Cyp2c9*2 and *3 and VKORC1 gene polymorphism. Indian J Med Res. 2013; 137(1):203-9.

10. Suarez-Kurtz G,Botton MR.Pharmacogenomics of Warfarin in populations of African Descent.British Journal of Clinical Pharmacology.2013; 75(2):334-46.

11. Owen RP, LiG.VKORC1 pharmacogenomics summary. Pharmacogenet Genomics.2010Oct; 20(10):642-4.

12. D’Andrea G,D'Ambrosio RL.Apolymorphism in the
VKORC1 gene is associated with an interindividual variability in the anticoagulant effect of warfarin. Blood.2005;105:645-9.

13. Dean L. Warfarintherapy and the genotype CYP2C9 and VKORC1. Medical Genetic Summaries. Bethesda(MD): National Centre for Biology Information (US); 2012.

14. Fung E, Belknap SM.Effect of genetic variants,especially CYP2C9 and VKORC1 in pharmacology of warfarin. Semin Thromb Hemost.2012;38(8):893-904. 